\title{
A comparative study of dynamic adsorption of anionic synthetic and nanocellulose-based surfactant in Malaysian reservoir
}

\author{
Muhammad Ayoub ${ }^{1}$. Mysara Eissa Mohyaldinn ${ }^{2}$. Syed Mohammad Mahmood ${ }^{2} \cdot$ Aqsha Aqsha $^{1}$. \\ Shiferaw Regassa Jufar ${ }^{2} \cdot$ Sarah Farrukh $^{3} \cdot$ Farrukh Inayat $^{4} \cdot$ Muhammad Rashid Shamsuddin $^{1}$
}

Received: 1 November 2018 / Accepted: 20 July 2019 / Published online: 24 August 2019

(c) The Author(s) 2019

\begin{abstract}
This project is aimed at studying the applicability of nanocellulose-based surfactant as a sustainable surfactant for enhanced oil recovery process (EOR) in Malaysia. Abundant biomass waste from palm oil industry in Malaysia is hard to be disposed of. Therefore, potential application of biomass in chemical EOR is an attractive alternative to minimize these problems. For this study, nanocellulose is synthesized from oil palm empty fruit bunch (OPEFB) and undergoes chemical modification for it to act like a surfactant. All methods and techniques in synthesizing nanocellulose and preparing nanocellulose-based surfactant are made inhouse. While waiting for the material preparation, adsorption study is carried out by using anionic synthetic surfactant. Characterization result shows the nanocellulose undergoes chemical modification successfully. The IFT results for the nanocellulose-based surfactants are also in a good and acceptable range, but there are some limitations in using nanocellulose-synthesized surfactant. The average particle size of nanocellulose is $283.5 \mu \mathrm{m}$ which is larger than size of the reservoir pore throat. Dynamic adsorption cannot be performed as the large particle size of nanocellulose might plug the porous domains. Therefore, it is recommended to further improve the method of synthesizing nanocellulose from OPEFB because the nanocellulose-based surfactant is expected to have potential of lower adsorption in porous media once it becomes nanosized and due to its advantages such as being of lower cost and environmental friendly compared to other commercial surfactants.
\end{abstract}

Keywords Nanocellulose $\cdot$ Surfactant . Dynamic adsorption $\cdot$ Chemical enhanced oil recovery

\section{Introduction}

Malaysia is well known among the largest producers and the exporters of Palm Oil in the world. Malaysia has more than 400 plan oil mills on roughly 5 million hectares of land (Board 2011; Bhat et al. 2018). In reality, palm oil production is only about $10 \%$, while the rest $90 \%$ are biomass waste. Several examples of palm waste are empty fruit bunch

Muhammad Ayoub

muhammad.ayoub@utp.edu.my

1 Chemical Engineering Department, Universiti Teknologi PETRONAS, 32610 Seri Iskandar, Perak, Malaysia

2 Petroleum Engineering Department, Universiti Teknologi PETRONAS, 32610 Seri Iskandar, Perak, Malaysia

3 National University of Sciences and Technology, H-12 Sector, Islamabad 44000, Pakistan

4 Exploration (Prospect Generation) Department, OGDCL, Islamabad, Pakistan
(OPEFB), palm kernel (OPK), palm fronds (OPF), and palm fiber (OPMF). It has been discovered that OPEFB has contained the highest cellulose compared to other waste stated (Board 2011; Bhat et al. 2018; Wei et al. 2016). This abundant biomass can be easily obtained at low price in Malaysia (Board 2011). Large amount of oil palm waste in Malaysia is hard to be disposed of. Therefore, potential application of biomass in chemical enhanced oil recovery (CEOR) is an alternative to minimize the operational problems.

Combined with the recent advancements in nanotechnology, a new type of green surfactant and cellulose extracted from OPEFB can be converted into nanosize and modified to act like surfactant primarily to reduce the interfacial tension (IFT) for CEOR purposes (Bryan and Kantzas 2007; Morsy 2014; Mishra et al. 2014; Iwamoto et al. 2008; Mohajeri et al. 2015). The application of surfactant in CEOR is not new, and the surfactant injection for oil recovery was started in the 1920s. In the early age of surfactant development, the reaction chemistry and process was not well understood, 
and chemicals were not feasible economically. Until 1980, the use of surfactants for EOR was considered as similar to "micellar/polymer" flooding (Chen et al. 2009; Beck-Candanedo et al. 2005). Later on, the term surfactant flooding was associated with ways to improve gas/steam flooding. Many surfactants are available in the market these days. The specific requirement of surfactants for CEOR purpose includes chemical and thermal compatibility at desired reservoir conditions, salinity tolerance, low adsorption, in porous media. Moreover, in some cases, surfactant did not manage to lower the interfacial tension to a level required and results in a higher adsorption in porous media (Azam et al. 2013; Cheraghian and Hendraningrat 2015; Dong et al. 1998).

One of the remarkable characteristic of nanocellulosebased surfactant is that it can easily pass through typical pore throats in reservoirs. Nanocellulose-based surfactant, which can be extracted from OPEFB, is also a biodegradable material (bio-nano) and low-cost alternative compared with current chemical surfactant (Han et al. 2009; Ghaedi et al. 2017; Karnanda et al. 2013). This is expensive and may contaminate the environment if left under reservoir. Despite the positivity, during surfactant flooding, physicochemical attraction among the surfactant and pore walls may still lead to significant adsorption and loss of surfactant in the reservoir, which is not economical (Neale et al. 1981; Liu et al. 2008; Hong et al. 2007).

In this study, for the purpose of evaluating the applicability of nanocellulose-based surfactant as a sustainable surfactant for EOR process in Malaysia, effluent-surfactant concentration was measured enabling the evaluation of nanocellulose-based surfactant adsorption by conducting dynamic adsorption test on Berea sandstone. This hopefully will help the oil producers in meeting high energy demand and reducing abundant waste from Palm Oil industry.

\section{Materials and methods}

\section{Materials}

The main materials for this research are oil palm empty fruit bunch, sodium dodecyl sulfate, synthetic anionic surfactant, and Berea sandstone core samples. Chemical composition of Berea sandstone is indicating in Table 1 obtained from $\mathrm{XRF}$ analysis.

\section{Apparatus and procedure}

The synthetic Berea sandstone samples were purchased from the market. Prior to the experiments, the cores are cleaned by using Soxhlet extractor with toluene and methanol. After this, the cores were dried at $50{ }^{\circ} \mathrm{C}$ in the oven
Table 1 Density of material for IFT test

\begin{tabular}{ll}
\hline Material & Density $\left(\mathrm{g} / \mathrm{cm}^{3}\right)$ \\
\hline Mineral oil & 0.863 \\
Brine & 1.020 \\
Nanocellulose-based surfactant & 1.0073 \\
Synthetic surfactant & 1.0240 \\
\hline
\end{tabular}

Table 2 IFT result

\begin{tabular}{lc}
\hline Sample & IFT $(\mathrm{mN} / \mathrm{m})$ \\
\hline Mineral oil + brine & 21.13 \\
Mineral oil + synthetic surfactant & 3.31 \\
Mineral oil + nanocellulose-based surfactant & 9.39 \\
\hline
\end{tabular}

for 2 days. After cleaning and drying, these core samples will undergo characterization to investigate their properties (Table 2).

1. Core Sample Characterization X-ray fluorescence spectroscopic (XRF) tests were conducted to investigate the composition of Berea core samples. Other than that, POROPERM is also conducted to obtain the properties of core samples. Other parameters such as sample length, diameter, and mass are also measured.

2. Brine Preparation Brine is prepared to represent the formation water inside reservoir. For this research, sodium chloride solution (brine) of $35,000 \mathrm{ppm}$ is used to represent the reservoir condition of salinity.

3. Synthetic Surfactant Preparation In this research study, while preparing for nanocellulose and nanocellulosebased surfactant, some extra work is done in which several commercialize surfactants are prepared to study their adsorption. These synthetic surfactants are prepared 10,000 ppm in 35,000 ppm brine.

4. Nanocellulose Preparation Empty fruit bunch (EFB) is used as the source for nanocellulose. There are several methods successfully in preparing cellulose fibril from cellulose fiber such as method recommended by Chen et al. (2009), Dong et al. (1998) and Beck-Candanedo et al. (2005). For this study, a method suggested by Iwamoto et al. (2008) is used in preparing cellulose fiber and nanofiber. For cellulose fiber, the method is as such:

(a) OPEFB is cut to small length

(b) Extractives such as resins, oil, fats and waxes are removed by using Soxhlet extractor by using an ethanol for approximately 2 days

(c) The extracted fibers then are soaked several times in sodium chlorite solution with $\mathrm{pH}$ of $4-5$ at $70{ }^{\circ} \mathrm{C}$ for $1 \mathrm{~h}$ before washed with deionized water. This process takes place in order to remove lignin. 
(d) Extraction of hemicellulose took place by bleaching the fibers using potassium hydroxide solution for 1 day before rinsing with deionized water until the $\mathrm{pH}$ is neutralized.

For cellulose nanofiber, the method is as such:

(a) $20 \mathrm{~g}$ of cellulose fibers extracted previously is hydrolyzed in $210 \mathrm{~mL}$ of sulfuric acid solution for approximately $2 \mathrm{~h}$ at $45^{\circ} \mathrm{C}$.

(b) Then $400 \mathrm{~mL}$ of cold water is added to terminate the hydrolysis process.

(c) The solution is centrifuged at 11,000 rpm for $30 \mathrm{~min}$, and the precipitate produces is re-suspended in water with strong agitation, followed by centrifugation. This process repeated until $\mathrm{pH}$ reached 5 .

(d) After that, dialysis is carried out to neutralize the $\mathrm{pH}$ to 7.

(e) The suspension produced was sonicated for half an hour.

(f) Lastly, the product is stored in a refrigerator for freezing.

The product from this pretreatment will undergo characterization and chemical modification to produce nanocellulose-based surfactant.

5. Nanocellulose-based Surfactant Preparation The extracted nanocellulose from the above experiments is now used in formulating the nanocellulose-based surfactant. The procedure for this experiment is based on research by Sagir et al. (2015), but nanocellulose is used:

(a) Mixed octadecylamine, extracted nanocellulose, and sodium dodecyl benzene sulfonate (SDBS) surfactant.

(b) 10,000 ppm brine is added to this mixture and warmed at $65^{\circ} \mathrm{C}$ and stirred for approximately 1 day. The blend is prepared at a level of $2 \%$ total active surfactant.

6. Nanocellulose and Nanocellulose-based Surfactant Characterization X-ray powder diffraction is carried out on the product extracted from EFB for the identification of crystalline materials (e.g., minerals and inorganic compounds). The nanocellulose is subjected to a powder form before being analyzed with D8 Pro Advanced XRD machine manufacturer by Bruker instrument. Other than that, particle size test is also run. The main objective is to check whether the product extracted from EFB is successfully converted into nanosized or not. Scanning electron microscope (SEM) is used to obtain information about the surface topography and composition. SEM is run for both nanocellulose extracted and nanocellulosebased surfactant. Fourier transform infrared spectroscopy (FTIR) is a technique which is used to obtain an infrared spectrum of absorption or emission of a solid, liquid, or gas (Rodriguez et al. 2009; Sodeifian et al. 2015; Srivastava et al. 2009). The FTIR is run on both nanocellulose extracted and nanocellulose-based surfactant.

7. Nanocellulose-based Surfactant and Synthetic Surfactant Interfacial Tension Test Interfacial tension for nanocellulose-based surfactant and synthetic surfactant is investigated by using pendant drop tensiometer. Interfacial tension of mineral oil versus $35,000 \mathrm{ppm} \mathrm{NaCl}$ with $1.0 \mathrm{wt} \%$ nanocellulose-based surfactant, $1.0 \mathrm{wt} \%$ synthetic surfactant. To run this test, density of material such as mineral oil, brine water, nanocellulose-based surfactant, and synthetic surfactant is measured by using density meter. The external phase for IFT test is mineral oil.

\section{Dynamic adsorption test}

The method of dynamic study applied in this project would be the effluent-surfactant concentration method measured by UV-visible spectrophotometer (Zhou et al. 2005; Yu et al. 2012; Zhou and Liu 2005). Benchtop permeability system (BPS) is used to perform core flooding or to inject surfactant into core. First, all core samples are saturated with $35,000 \mathrm{ppm}$ brine for approximately $24 \mathrm{~h}$. The pump is switched on for $30 \mathrm{~min}$ and left for approximately $24 \mathrm{~h}$ inside the vacuum pump. The weight of core samples is recorded. After installing the core into the core holder, formation brine, $35,000 \mathrm{ppm}$ brine, is injected into the core sample at $1.0 \mathrm{cc} / \mathrm{min}$. Accuracy of pump rate is measured to ensure that it is working effectively. Brine is injected for $300 \mathrm{ml}$ to ensure the core is $100 \%$ saturated with brine to achieve steady-state condition (pressure is constant) and also cleaned from any chemicals. Before injecting surfactant, brine solution come out or produce from the system must be a clear solution. Surfactant is injected after clear brine produce. Sample is collected for every $0.15 \mathrm{PV}$ until it reached $6 \mathrm{PV}$. Then, $100 \mathrm{ml}$ secondary brine is injected. The samples collected are centrifuged at 3,700 rpm for $30 \mathrm{~min}$. The supernatant is filtered out from each sample by using a syringe filter. Then sample of $0.151 .5 \mathrm{PV}, 1.5-2.25 \mathrm{PV}$, and 2.25-6 are diluted 3, 4 , and 5 times, respectively. The process is repeated for each surfactant involved in this dynamic study. Before the concentration for each sample can be determined, a calibration graph for each surfactant needs to be established. Calibration graph is established by measuring the absorbance of $100 \mathrm{ppm}, 200 \mathrm{ppm}, 300 \mathrm{ppm}, 400 \mathrm{ppm}, 500 \mathrm{ppm}$, $600 \mathrm{ppm}, 700 \mathrm{ppm}, 800 \mathrm{ppm}, 900 \mathrm{ppm}$, and 10,000 ppm for each surfactant with reference to $35,000 \mathrm{ppm}$ brine as base. The accuracy of calibration graph is very important. R-squares value must be close to 1 . After calibration graph for respective surfactant is established, the

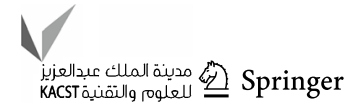


absorbance for each sample is measured, and then the concentration can be calculated from the trend of calibration graph. Adsorption of the surfactant is calculated by using the following equation:

$q e=\frac{V(C i-C e)}{M}$

where $q e$ is adsorption of adsorbent $(\mathrm{mg} / \mathrm{g}), C i$ initial concentration $(\mathrm{mg} / \mathrm{L}), C e$ equilibrium concentration $(\mathrm{mg} / \mathrm{L}), V$ volume of the solution (L), and $M$ mass of the adsorbent used $(\mathrm{g})$.

\section{Results and discussion}

\section{X-ray powder diffraction}

XRD result shows for nanocellulose a single high intensity at $23^{\circ}$, which suggests that the depolymerization of hemicellulose and delignification has been successfully achieved. The sharp peak also describes the crystalline nature of the sample similar to a result reported earlier by 7 (Iwamoto et al. 2008). The degree of the crystallinity for the nanocellulose samples was determined by using the following equation:

Crystallinity $(\%)=\left(1-\frac{I_{\mathrm{AM}}}{I_{002}}\right) \times 100 \%$

$I_{002}$ maximum intensity of the peak, $I_{\mathrm{AM}}$ second maximum intensity of the peak.

The XRD of the nanocellulose extracted from OPEFB were discussed according to the diffraction peaks from "Appendix"-XRD diffraction results. From the figure, we can notice that there are two sharp peaks at $2 \theta=22^{\circ}$ and $2 \theta=15^{\circ}$. Therefore, the nanocellulose extracted from OPEFB results in $58.33 \%$ crystallinity of cellulose, which shows an excellent extraction of cellulose.

\section{Particle size of nanocellulose}

The mean size for nanocellulose produced is $283.5 \mu \mathrm{m}$. It is expected for the product to be in nanosize which is in range of $20 \mathrm{~nm}$ or $0.02 \mu \mathrm{m}$. The method or technique used for nanocellulose preparation for this study did not manage to successfully convert cellulose into nanosized. More research and time are still needed to make the cellulose
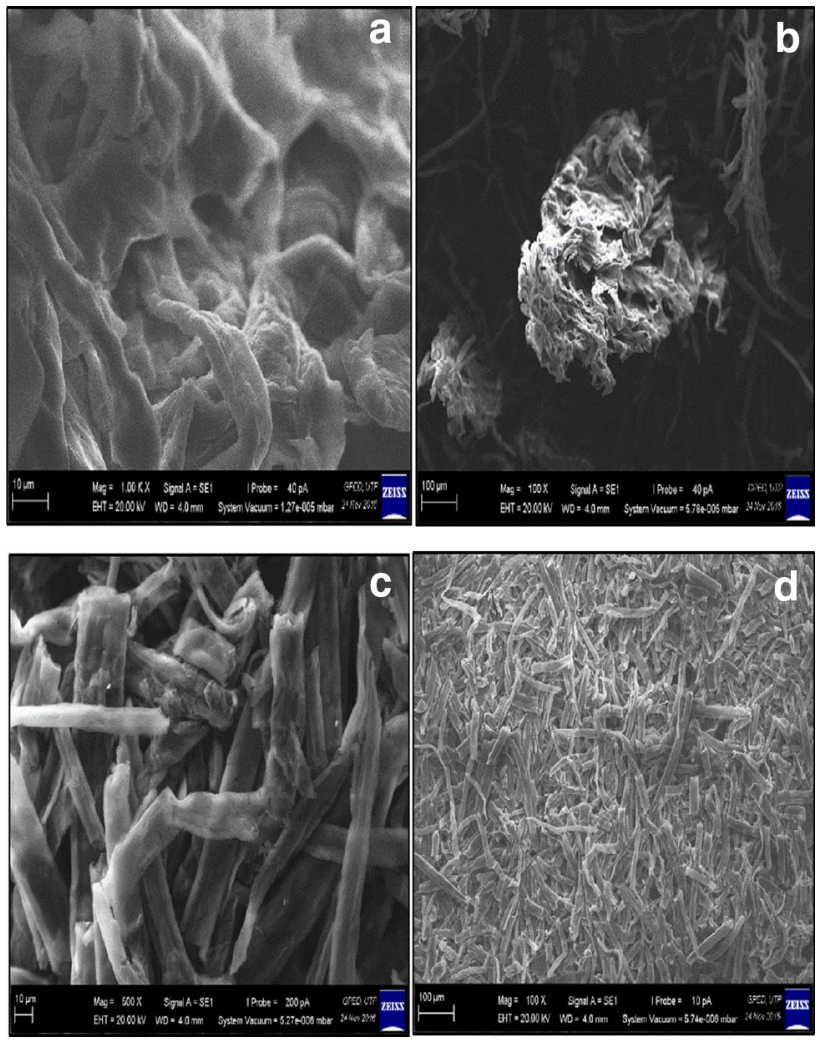

Fig. 1 a, b SEM images of nanocellulose, c, d SEM images for nanocellulose-based surfactant

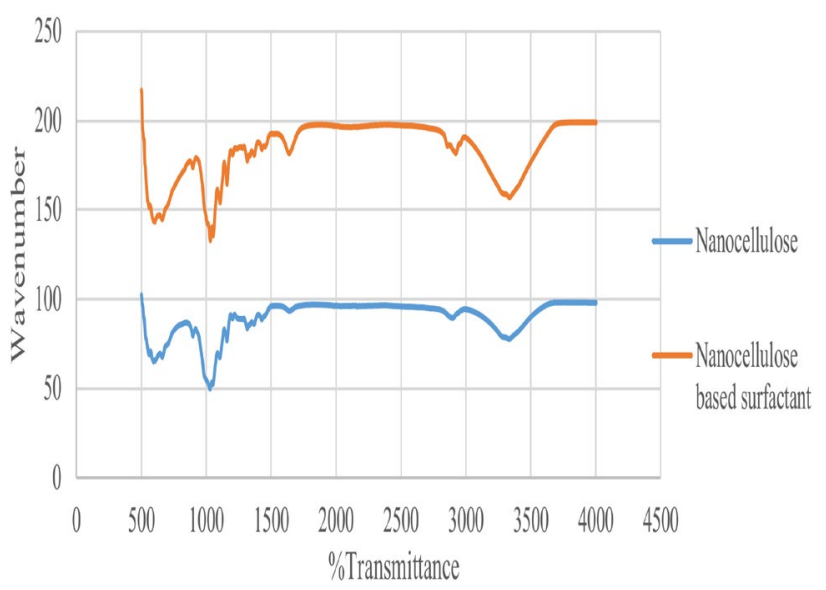

Fig. 2 FTIR of nanocellulose and nanocellulose-based surfactant

successful to reach nanosize, but it is possible (Isogai 2012). Figure 1 shows scanning electron microscope 
(SEM) result for nanocellulose and nanocellulose-based surfactant.

From SEM result, it is can be observed that the nanocellulose is to be coagulated, while for nanocellulose-based surfactant, it can be seen that the surface topography has changed. The preparation method for nanocellulose-based surfactant successfully changed coagulated nanocellulose to be more disperse.

\section{Fourier transform infrared spectroscopy (FTIR)}

The result infrared spectroscopy for nanocellulose and nanocellulose-based surfactant is shown in Fig. 2.

From the infrared spectroscopy, IR spectra of nanocellulose were obtained; visually, there is no difference between spectrum of nanocellulose and nanocellulose-based surfactant. The strong and broad bands at $3332 \mathrm{~cm}^{-1}$ (nanocellulose) and $3335 \mathrm{~cm}^{-1}$ (nanocellulose-based surfactant) indicate the characteristic of $\mathrm{OH}$ group or phenolic compound. The bands at $2996 \mathrm{~cm}^{-1}$ (nanocellulose) and $2922 \mathrm{~cm}^{-1}$ (nanocellulose-based surfactant) are due to the bending of vibration in phenolic $\mathrm{OH}$ group, while the bands at $1029 \mathrm{~cm}^{-1}$ (nanocellulose) and $1032 \mathrm{~cm}^{-1}$ (nanocellulose-based surfactant) are characteristic of primary alcohol. From the above result, it shows that the extracted nanocellulose contained hydroxyl

Table 3 Component of Berea sandstone

Table 4 Core parameter

\begin{tabular}{ll}
\hline Component & Amount (wt\%) \\
\hline $\mathrm{SiO} 2$ & 60.8 \\
$\mathrm{~A} 12 \mathrm{O} 3$ & 23.8 \\
$\mathrm{Fe} 2 \mathrm{O} 3$ & 7.1 \\
$\mathrm{~K} 2 \mathrm{O}$ & 3.8 \\
$\mathrm{P} 2 \mathrm{O} 5$ & 1.4 \\
$\mathrm{MgO}$ & 1.2 \\
$\mathrm{TiO} 2$ & 0.9 \\
$\mathrm{CaO}$ & 0.6 \\
\hline
\end{tabular}

$(\mathrm{O}-\mathrm{H})$ and carbonyl $(\mathrm{C}=\mathrm{O})$ functional group that can contribute to stable surfactant formulation.

\section{Interfacial tension}

Density of nanocellulose-based surfactant measured by density meter:

The result of IFT is as such with mineral oil as external phase:

From the IFT result, the nanocellulose-based surfactant indicates good reduction in interfacial tension.

$X$-Ray Fluorescence Spectroscopic Composition of core sample is given in Table 3 below:

The surface chemistry for the core sample depends on silica and aluminum since they have the highest fractions. Four core samples are used in the experiment. POROPERM results are given in Table 4 , where $K_{\text {air }}$ represents permeability of the air and $\mathrm{k}_{\infty}$ represents average permeability.

Before running the core flooding, the BPS pump is checked for accuracy. At setting rate of $1 \mathrm{cc} / \mathrm{min}$, the pump gives out $4 \mathrm{ml}$ for $4 \mathrm{~min}$. This test is repeated three times, and the result is the same. This indicates the accuracy of the pump. After preflushing the core samples with brine, at some early time, the product that came out is not a clear solution; yellowish in color indicates that in the core there are other chemical substances. It is suspected for the yellowish solution is to be toluene. After some time, a constant clear solution came out which indicates brine water and means the core is fully saturated

Fig. 3 Image for IFT test for nanocellulose-based surfactant

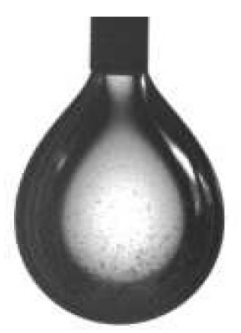

\begin{tabular}{lllll}
\hline Parameter & Sandstone \#1 & Sandstone \#2 & Sandstone \#3 & Sandstone \#4 \\
\hline Core ID & $\mathrm{Z} 1$ & $\mathrm{Z} 2$ & $\mathrm{Z} 3$ & $\mathrm{Z} 5$ \\
Length $(\mathrm{cm})$ & 6.50 & 7.37 & 6.24 & 7.23 \\
Diameter $(\mathrm{cm})$ & 3.81 & 3.76 & 3.77 & 3.74 \\
Mass $(\mathrm{g})$ & 164.974 & 182.242 & 151.567 & 177.392 \\
$\mathrm{~K} \infty(\mathrm{md})$ & 29.54 & 51.06 & 44.91 & 22.70 \\
$K_{\text {air }}(\mathrm{md})$ & 32.52 & 55.27 & 48.80 & 25.26 \\
Porosity $(\%)$ & 22.32 & 19.14 & 20.43 & 20.03 \\
Pore volume $\left(\mathrm{cm}^{3}\right)$ & 16.92 & 15.67 & 14.23 & 15.91 \\
\hline
\end{tabular}




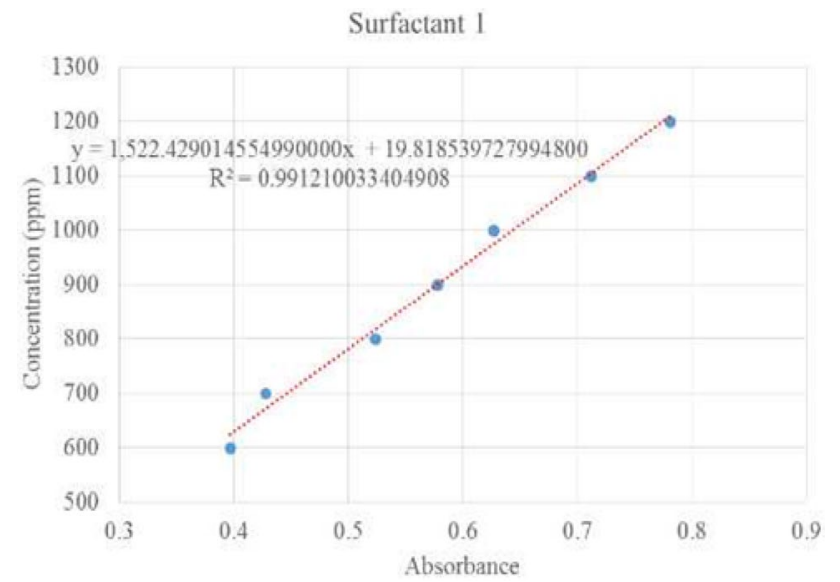

Fig. 4 Calibration curve for surfactant 1

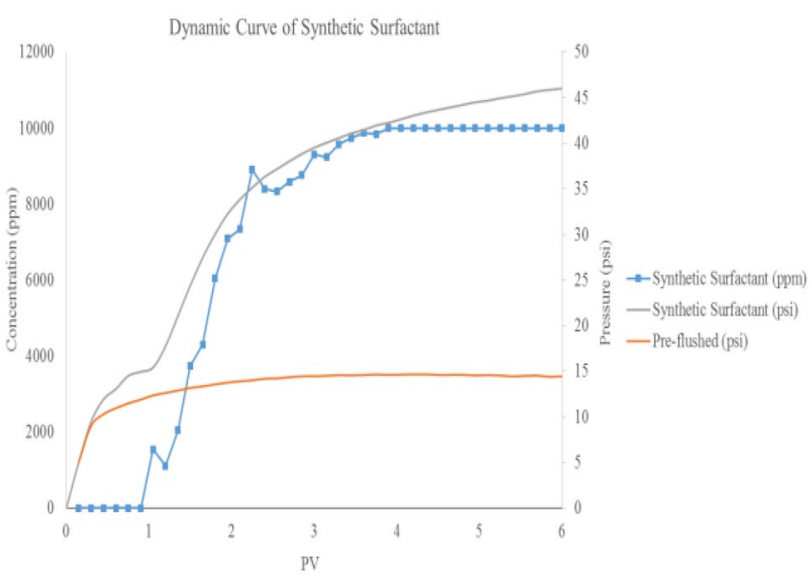

Fig. 5 Dynamic adsorption curve for surfactant 1

with brine. Calibration graph of surfactant 1 is measured by using UV spectrometer. The peak of surfactant 1 is $240 \mathrm{~nm}$ (Figs. 3, 4).

In real situation of surfactant flooding, adsorption that occurs onto the reservoir rocks is dynamic. Thus, a dynamic adsorption method gives more practical and better description on the real situation and the result from it is more reliable.
Figure 5 shows the dynamic adsorption curve of surfactant 1 and also the pressure difference during surfactant flooding and pre-flushing. From the figure, it can be observed that the presence of surfactant is detected after $1 \mathrm{PV}$. Before 1PV, it is purely brine. There are differences in pressure during surfactant flooding (grey line) and pressure during preflushing (orange line). That indicates that the surfactant controls the mobility factor. The surfactant reached equilibrium at 4PV. The adsorption results were determined by the decrease in surfactant concentration from the area integration during the stabilization process. The adsorption for the surfactant 1 is $4.81467 \mathrm{mg} / \mathrm{g}$.

\section{Conclusion}

Dynamic adsorption of an anionic synthetic surfactant is successfully carried out, but the study of dynamic adsorption of nanocellulose-based surfactant cannot be proceeded due to some drawbacks in the particle size during synthesis of the nanocellulose. There were several bundles of fibril aggregates after the acid hydrolysis. The number of bundles of fibril aggregate can be decreased due to longer test duration for acid hydrolysis time. It is recommended that in order for the cellulose fibers to be obtained at nanosize, longer acid hydrolysis process is needed. Overall, the nanocellulose shows good result in lowering the IFT. Therefore, it is recommended to further study the potential of nanocellulose in chemical enhanced oil recovery with another method in converting the cellulose into nanosize. This is because the advantages of nanocellulose such as nanosized, lower cost and environmental friendly is a promising alternative for synthetic surfactant currently used in petroleum industry. It could overcome some limitation of the existing surfactant. Besides, we can contribute to producing a new green solution, potentially without impacting the environment.

Acknowledgements Author wants to acknowledge to Universiti Teknologi PETRONAS and MOHE for providing found for research.

Open Access This article is distributed under the terms of the Creative Commons Attribution 4.0 International License (http://creativeco mmons.org/licenses/by/4.0/), which permits unrestricted use, distribution, and reproduction in any medium, provided you give appropriate credit to the original author(s) and the source, provide a link to the Creative Commons license, and indicate if changes were made.

\section{Appendix}

See Fig. 6. 


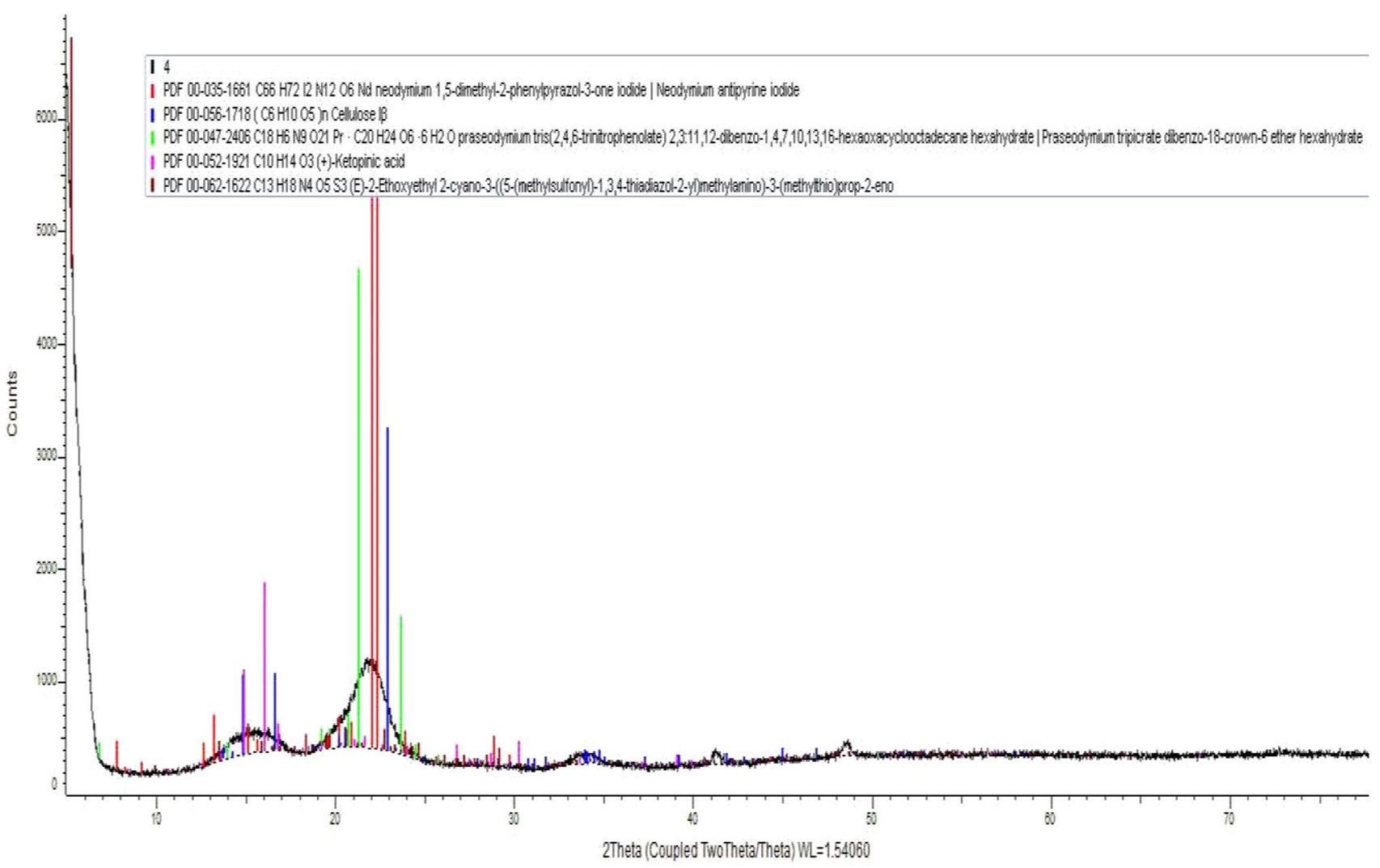

Fig. $6 \mathrm{X}$-ray powder diffraction result for the nanocellulose

\section{References}

Azam MR, Tan IM, Ismail L, Mushtaq M, Nadeem M, Sagir M (2013) Static adsorption of anionic surfactant onto crushed Berea sandstone. J Pet Explor Prod Technol 3(3):195-201. https://doi. org/10.1007/s13202013-0057-y

Beck-Candanedo S, Roman M, Gray DG (2005) Effect of reaction conditions on the properties and behavior of wood cellulose nanocrystal suspension. Biomacromol 6:1048-1054

Bhat AH, Rehman WU, Khan IU, Khan I, Ahmad S, Ayoub M, Usmani MA, (2018) Nanocomposite membrane for environmental remediation. Polymer-based Nanocomposites for Energy and Environmental Applications, 407-440

Board MPO (2011) Malaysian Palm Oil Industry. Retrieved 20 June 2016, 2016, from http://www.palmoilworld.org/about_malaysiani ndustry.html

Bryan J, Kantzas A (2007) Enhanced heavy-oil recovery by alkali-surfactant flooding. Published by Society of Petroleum Engineering

Chen Y, Liu C, Chang PR, Cao X, Anderson DP (2009) Bionanocomposites based on pea starch and cellulose nanowhiskers hydrolyzed from pea hull fibre: effect of hydrolysis time. Carbohydr Polym 76:607-615

Cheraghian G, Hendraningrat L (2015) A review on applications of nanotechnology in the enhanced oil recovery part B: effects of nanoparticles on flooding. Int Nano Lett 6(1):110. https://doi. org/10.1007/s40089-015-0170-7
Dong XM, Revol JF, Gray DG (1998) Effect of microcrystallite preparation conditions on the formation of colloid crystals of cellulose. Cellulose 5:19-32

Ghaedi H, Ayoub M, Sufian S, Murshid G, Farrukh S, Shariff AM (2017) Investigation of various process parameters on the solubility of carbon dioxide in phosphonium-based deep eutectic solvents and their aqueous mixtures: experimental and modeling. Int J Greenhouse Gas Control 66:147-158

Han C, Delshad M, PopeGA, Sepehrnoori K (2009) Coupling equationof-state compositional and surfactant models in a fully implicit parallel reservoir simulator using the equivalent-alkanecarbonnumber concept. Published by Society of Petroleum Engineering

Hong P, Fa C, Wei Y, Sen Z (2007) Surface properties and synthesis of the cellulose-based amphoteric polymeric surfactant. Carbohyd Polym 69(4):625-630, ISSN 0144-8617

Isogai A (2012) Preparation and characterization of TEMPO-oxidized cellulose nanofibers. Bulletin of the Tokyo University Forests 126: 1-47. In press http://hdl.handle.net/2261/5146

Iwamoto S, Abe K, Yano H (2008) The effect of hemicelluloses on wood pulp nanofibrillation and nanofiber network characteristics. Biomacromol 9:1022-1026

Karnanda W, Benzagouta MS, AlQuraishi A, Amro MM (2013) Effect of temperature, pressure, salinity, and surfactant concentration on IFT for surfactant flooding optimization. Arab J Geosci $6: 3535-3544$ 
Liu S et al (2008) Favorable attributes of alkaline-surfactant- polymer flooding. SPE J 13(01):516

Mishra PC, Mukherjee S, Kumar Nayak S, Panda A (2014) A brief review on viscosity of nano fluids. J Int Nano Lett 4:109-120

Mohajeri M, Hemmati M, Shekarabi AS (2015) An experimental study on using a nanosurfactant in an EOR process of heavy oil in a fractured micromodel. J Petrol Sci Eng 126:162-173. https://doi. org/10.1016/j.petrol.2014.11.012

Morsy SMI (2014) Role of surfactants in nanotechnology and their applications. Int J Curr Microbiol App Sci 3(5):237260

Neale G, Hornof V, Chiwetelu C (1981) Importance of lignosulfonates in petroleum recovery operations. J Chem 59(13):1938-1943

Rodriguez E, Roberts MR, Yu H, Huh H, Bryant SL (2009) Enhanced migration of surface-treated nanoparticles in sedimentary rocks. SPE Annual Technical Conference and Exhibition, New Orleans, Louisiana

Sagir M, Tan IM, Mushtaq M, Pervaiz M, Tahir MS (2015) Synthesis and CO2 EOR applications of foaming surfactants. J Pet Environ Biotechnol 7:257. https://doi.org/10.4172/2157-7463.1000257

Sodeifian G, Daroughegi R, Aalaie J (2015) Study of adsorptive behavior of sulfonated polyacrylamide onto carbonate rock particles to enhance oil recovery. Korean J Chem Eng 32(12):2484-2491. https://doi.org/10.1007/s11814-015-01120
Srivastava M, Zhang J, NyugenQP, Pope GA (2009) A systematic study of alkaline-surfactant-gas injection as an EOR technique. Published by Society of Petroleum Engineering

Wei B, Li Q, Jin Fayang, Li Hao, Wang Chongyang (2016) The potential of a novel nanofluid in enhancing oil recovery. Energy Fuels 30:9

Yu J, An C, Mo D, Liu N, Lee RL (2012) Study of adsorption and transportation behavior of nanoparticles in three different porous media. Soc Pet Eng 1:1-3. https://doi.org/10.2118/153337-ms

Zhou W, Liu MDQ (2005) Experimental investigation of surfactant adsorption on sand and oil-water interface in heavy oil/water/sand systems. Pet Soc J

Zhou W, Dong M, Liu Q, Xiao H (2005) Experimental investigation of surfactant adsorption on sand and oil-water interface in heavy oil/water/sand systems. Pet Soc Canada. https://doi. org/10.2118/2005-1

Publisher's Note Springer Nature remains neutral with regard to jurisdictional claims in published maps and institutional affiliations. 